\title{
Post-Anesthesia Hookwire Localization of Pulmonary Ground Glass Nodules Guided by Computed-Tomography Three-Dimensional Reconstruction: A Feasibility Study
}

\section{Chong Wang}

Beijing Chest Hospital https://orcid.org/0000-0001-7278-6119

Yang Liu

Beijing Chest Hospital

Bo Xiao

Beijing Chest Hospital

Shuku Liu (D liushuku@aliyun.com)

Beijing Chest Hospital

\section{Research}

Keywords: ground glass nodules, three-dimensional reconstruction, video assisted thoracoscopic surgery, localization, post-anesthesia

Posted Date: March 19th, 2021

DOI: https://doi.org/10.21203/rs.3.rs-332644/v1

License: (1) (i) This work is licensed under a Creative Commons Attribution 4.0 International License.

Read Full License 


\section{Abstract}

Background: Computed-tomography(CT) guided hookwire localization has disadvantages, such as pain, patient transport risk and increased radiation exposure. In order to overcome the above shortcomings, our method is post-anesthesia hookwire localization guided by preoperative CT three-dimensional reconstruction. The effectiveness and safety of this method will be studied.

Methods: A total of 36 patients(36 nodules) with ground glass nodules were consecutively included in this study. Three dimensional reconstruction of chest CT was performed to simulate the puncture path. The puncture point, the depth and angle of needle insertion were recorded. After anesthesia, the puncture point was marked on the patient's body surface. Hookwire was placed according to the previous planned puncture direction and depth. The effectiveness and complications of the localization were evaluated during the operation.

Results: Hookwire dislocation was found in 1 patient in operation. The other 35 nodules were successfully positioned. The median distance between hookwire and lesion was $14 \mathrm{~mm}(3-25 \mathrm{~mm})$. The effective rate was $97.2 \%(35 / 36)$. Only one patient found a small amount of blood in the chest. There was no pulmonary hematoma, blood pressure or oxygen decreasing in all patients. The complication rate was $2.8 \%(1 / 36)$ and the severe complication was 0 . The median time for localization was 3 minutes.

Conclusion: Post-anesthesia hookwire localization guided by preoperative three-dimensional reconstruction is a simple, safe and effective localization method, which is suitable for appropriate nodules, and is an important supplement to CT guided localization.

Trial registration: ChiCTR1900023853. Registered in Chinese Clinical Trail Registry on 14 June 2019, http://www.chictr.org.cn/showprojen.aspx?proj=40060

\section{Background}

Accurate localization of ground glass nodules during thoracoscopic surgery is important. At present, the most commonly used localization method is to embedding localizer(hookwire, microcoil, cyanoacrylate or indocyanine green) under computed tomography(CT) guidance before operation(1-4). These are accurate methods, but risks, such as bleeding, pneumothorax, pain and increased radiation exposure, should not be ignored, because the localization was performed in the CT room and the surgery may start after a few hours. At the same time, it takes up residents much extra time to contact the radiology department before operation, especially in larger hospitals.

Therefore, in order to overcome the above shortcomings, we carried out this study. Hookwire localization, guided by preoperative three-dimensional(3D) reconstruction, was performed after anesthesia. Now we report 36 cases localization and aim to examine the feasibility in clinical application.

\section{Methods}


This is a prospective cohort study without control group. Inclusion criteria: (1) patients scheduled for thoracoscopic lung surgery. (2) On thin slice CT, the lesions was pure ground glass nodules or non-pure ground glass nodules with solid components $\leq 50 \%$. (3) Patients agreed to participate in the study and signed informed consent. Exclusion criteria: (1) patients with pneumothorax and pleural effusion, unable to perform lung puncture; (2) patients' lesion covered by scapula; (3) large blood vessels around the lesions; (4) lesions are close to pleura and pleural traction sign may be directly seen during operation.

\section{D reconstruction before operation}

The DICOM format CT image files were imported into software Materialise Mimics (Leuven, Belgium, version: 21.0.0.406). The lesions, ribs and chest skin were reconstructed. The route of needle insertion was planned, which was led from the lesion to the skin surface, avoiding ribs, and perpendicular to the skin tangent line as far as possible. The intersection point with the skin was the puncture point. Taking the ipsilateral nipple and midline as reference in reconstructed skin, the location of inserting point, number of intercostal, direction and depth were recorded(Figure 1).

\section{Localization Procedure after anesthesia}

The patient was placed in a supine position after general anesthesia, and the nurse raised the patient's arm consistent with the position of preoperative CT scanning. Find the corresponding intercostal and mark puncture points referred by nipple and midline. Insert the hookwire(Argon Medical Devices Inc, USA) into the subcutaneous and intercostal muscle after disinfection. At this time, let the anesthesiologist inflate the lung and maintain it. Then put the needle into the lung according to the predetermined angle and depth, release the hookwire and pull out the cannula. Return to mechanical ventilation and put the patient in the operation position.

\section{Effectiveness and complication evaluation}

Effectiveness and complication of localization was evaluated in video assisted thoracoscopy surgery(VATS). Wedge resection or segmentectomy was performed around hookwire. If the lesion was found in the specimen, it was regarded as successful localization. If there was no lesion or hookwire dislocation, it was considered as failure, and then segmentectomy or lobectomy should be continued. The diagnosis of complications is based on whether there is bleeding, pulmonary hematoma, pericardial injury, or changes of vital signs on monitor when entering the chest.

\section{Statistical Analysis}

All data were recorded and analyzed in SPSS(version 23.0; IBM SPSS Inc, Armonk, NY).

\section{Results}

Between April 2020 and October 2020, 36 patients with 36 ground glass nodules(GGN) were actually participated in the study. Patients' baseline and nodule characteristics are summarized in Table 1. 
Table 1. Patients' baseline and nodules characteristics

\begin{tabular}{|ll|}
\hline Variables & $\mathrm{N}$ \\
\hline Gender, female/male & $28 / 8$ \\
\hline Median age & $55(27 \sim 77)$ \\
\hline CT features of lesions & \\
\hline mGGN & 6 \\
\hline pGGN & 30 \\
\hline Median size(cm) & $0.8(0.5 \sim 1.3)$ \\
\hline Median distance between pleura and lesion(cm) & $2.0(0.7 \sim 2.8)$ \\
\hline Lobe & \\
\hline RUL & 16 \\
\hline RML & 4 \\
\hline RLL & 6 \\
\hline LUL & 8 \\
\hline LLL & 2 \\
\hline
\end{tabular}

mGGN: Mixed ground glass nodules; pGGN: pure ground glass nodules; RUL: right upper lobe; RML: right middle lobe; RLL: right lower lobe; LUL: left upper lobe; LLL: left lower lobe.

Only one patient had a small amount of hemothorax, the volume was about $20 \mathrm{ml}$, and there was no active bleeding at the puncture site. No pulmonary hematoma, blood pressure and oxygen decreasing were found in all 36 patients. Therefore, the slight complication rate was $2.8 \%(1 / 36)$ and the severe complication was 0 (Table2).

Table 2. Localization evaluation and surgical results 


\begin{tabular}{|ll|}
\hline Variables & $\mathrm{N}$ \\
\hline Insertion direction & \\
\hline Perpendicular to skin & 26 \\
\hline Oblique insertion & 10 \\
\hline Median time consuming of localization(min) & $3(1 \sim 5)$ \\
\hline Median distance between hookwire and lesion(mm) & $14(3 \sim 25)$ \\
\hline Median distance between nodules and resection margins(mm) & $22(15 \sim 28)^{\star}$ \\
\hline Slight complication & 1 \\
\hline Resection range & \\
\hline Wedge resection & 23 \\
\hline Segmentectomy & 12 \\
\hline Lobectomy & 1 \\
\hline Touchable & \\
\hline Yes & 7 \\
\hline No & 29 \\
\hline Successful localization & 35 \\
\hline Pathology & 24 \\
\hline AlS & 8 \\
\hline MIA & \\
\hline Adenocarcinoma & \\
\hline
\end{tabular}

AIS: adenocarcinoma in situ; MIA: minimally invasive adenocarcinoma *patients with wedge resection were measured.

There was one hookwire dislocation when entering the chest. Because the palpation was not successful, lobectomy was performed. The lesion was found in the resected lobe, and the pathology was invasive adenocarcinoma. No dislocation was found in the remaining 35 patients. Under the hookwire guidance, wedge resection was performed in 23 cases and segmentectomy in 12 cases. The mean distance between hookwire and lesion was $14 \mathrm{~mm}(3-25 \mathrm{~mm})$. The effective rate of localization was $97.2 \%$ $(35 / 36)$. 
The median time for localization was 3 minutes (1-5 minutes) and 7 minutes (4-13 minutes) for preoperative 3D reconstruction. There were no perioperative complications, such as bleeding, embolism, arrhythmia, respiratory failure or chylothorax.

During the operation, $80.5 \%$ (29/36) of GGN were negative by palpation. Postoperative pathology: 4 GGN was adenocarcinoma in situ, 24 were microinvasive adenocarcinoma, and 8 were invasive adenocarcinoma. The latest postoperative staging was T1bNOMO.

\section{Discussion}

Some scholars have proposed methods that do not rely on CT guided lung puncture positioning, such as the use of intrathoracic natural anatomical markers, intraoperative ultrasound, 3D printing navigational template, intraoperative virtual reality, magnetic navigation/virtual bronchoscope(5-12). These methods reduce the risk of puncture, but the economic and time cost for latter several methods are too expensive to implement. The localization introduced in this study is not only an effective method but can reduce the risk of puncture and patient's painfulness as far as possible because the positioning procedure was performed in the operating room and after anesthesia. In addition, this method can decrease the radiation exposure of patients and the workload of doctors as much as possible.

The most important points of this study is to ensure that the localization is accurate and effective. The theoretical hypothesis is that localization is not biopsy and we only need to put the hookwire close to GGN to indicate the scope of the lesion. The following points are the key steps for an accurate localization. (1) The planned puncture point should be close to the anatomic landmarks of the body surface, such as nipple, midline, outer edge of breast and vertical line of nipple. The direction of puncture should be perpendicular to the skin section. The reason of the failure localization is that the designed route is oblique, which makes the angle difficult to simulate. (2) Make sure that the intercostal is correct. Generally, the first intercostal space is under the clavicle, and the sternal angle corresponds to the second rib. It is also could be confirmed by the distance from the nipple and the midline. (3) During puncture, pay attention to maintain the same body position as the preoperative CT scanning, such as keeping the upper limbs elevated.

In 2017 and 2019, Chen C., et al reported 3D printing navigational templates localization $(9,11)$. The principle is similar to our method. The position and direction of puncture are determined by navigational templates covered on patients' body. The median error between hookwire and the center of lesion is 10 $\mathrm{mm}$. The effectiveness and safety of navigational templates localization are perfect. But the procedure is still performed in CT room, which is difficult to use on a large scale because of its high economic and time cost.

This study has certain limitations. Firstly, not all GGN localization are suitable for this method, because puncture can only be performed in supine position. When the patients stay on the side or prone position, the GGN location is different from that of preoperative CT, which cannot guarantee the accuracy. Secondly, it cannot be evaluated after localization, which can only be verified during operation. If the 
localization fails and the lesion is untouchable, lobectomy or segmentectomy may be required. Finally, the number of cases is small. This is only a feasibility study, which needs more cases to verify.

\section{Conclusion}

Post-anesthesia hookwire localization guided by preoperative three-dimensional reconstruction is a simple, safe and effective localization method, which is suitable for appropriate nodules, and is an important supplement to CT guided localization.

\section{Abbreviations}

CT: computed-tomography

3D: three-dimensional

GGN: ground glass nodule

VATS: video assisted thoracoscopy surgery

\section{Declarations}

\section{Ethics approval and consent to participate}

This study has been approval by the clinical ethics committee of Beijing Chest Hospital. Written consent was obtained from all patients for the use of their records and imaging in future studies.

\section{Consent for publication}

Written informed consent for publication of their clinical details and clinical images was obtained from the patients. A copy of the consent form is available for review by the Editor of this journal.

\section{Availability of data and materials}

The datasets used and analyzed during the current study are available from the corresponding author on reasonable request.

\section{Competing interests}

The authors declare that they have no competing interests.

\section{Funding}

This study was fund by Beijing Tongzhou High Level Talent Development Support Plan (YHBJ2020001) and Beijing Municipal Administration of Hospitals Incubating Program(PX2021060). They provided materials and language embellishment support for this study 


\section{Authors' contributions}

CW designed the study and was a major contributor in writing the manuscript. YL was in charge of 3D reconstruction and GGN localization. BX contribute to collate and analyze research data. SL is responsible for quality control and GGN resection. All authors have read and approved the final manuscript.

\section{Acknowledgements}

Not applicable

\section{References}

1. Sui X, Zhao H, Yang F, Li JL, Wang J. Computed tomography guided microcoil localization for pulmonary small nodules and ground-glass opacity prior to thoracoscopic resection. $\mathrm{J}$ Thorac Dis. 2015;7(9):1580-7.

2. Wang C, Liu Y, Yang L, Liu SK. [Effectiveness and safety of CT-guided percutaneous intrapulmonary injection of indocyanine green for localization of pulmonary nodules and ground glass opacity]. Zhonghua Yi Xue Za Zhi. 2020;100(7):538-40.

3. Yao F, Yao J, Xu L, Wang J, Gao L, Wang J. Computed tomography-guided cyanoacrylate localization of small pulmonary nodules: feasibility and initial experience. Interact Cardiovasc Thorac Surg. 2019;28(3):387-93.

4. Zhao G, Yu X, Chen W, Geng G, Li N, Liu H, et al. Computed tomography-guided preoperative semirigid hook-wire localization of small pulmonary nodules: 74 cases report. J Cardiothorac Surg. 2019;14(1):149.

5. Abbas A, Kadakia S, Ambur V, Muro K, Kaiser L. Intraoperative electromagnetic navigational bronchoscopic localization of small, deep, or subsolid pulmonary nodules. J Thorac Cardiovasc Surg. 2017;153(6):1581-90.

6. Fang HY, Chang KW, Chao YK. Hybrid operating room for the intraoperative CT-guided localization of pulmonary nodules. Ann Transl Med. 2019;7(2):34.

7. Kim TH, Park CM, Lee SM, McAdams HP, Kim YT, Goo JM. Percutaneous transthoracic localization of pulmonary nodules under C-arm cone-beam CT virtual navigation guidance. Diagn Interv Radiol. 2016;22(3):224-30.

8. Mao R, Shi W, Chen D, Kadeer XM, Li M, Fei K, et al. Technique of skeletal navigation for localizing solitary pulmonary nodules. J Surg Oncol. 2017;116(6):763-5.

9. Sun W, Zhang L, Wang L, Ren Y, She Y, Su H, et al. Three-Dimensionally Printed Template for Percutaneous Localization of Multiple Lung Nodules. Ann Thorac Surg. 2019;108(3):883-8.

10. Yang SM, Lin CK, Chen LW, Chen YC, Huang HC, Ko HJ, et al. Combined virtual-assisted lung mapping (VAL-MAP) with CT-guided localization in thoracoscopic pulmonary segmentectomy. Asian J Surg. 2019;42(3):488-94. 
11. Zhang L, Li M, Li Z, Kedeer X, Wang L, Fan Z, et al. Three-dimensional printing of navigational template in localization of pulmonary nodule: A pilot study. J Thorac Cardiovasc Surg. 2017;154(6):2113-9. e7.

12. Zhou Z, Wang Z, Zheng Z, Cao J, Zhang C, He Z, et al. An "alternative finger" in robotic-assisted thoracic surgery: intraoperative ultrasound localization of pulmonary nodules. Med Ultrason. 2017;19(4):374-9.

\section{Figures}

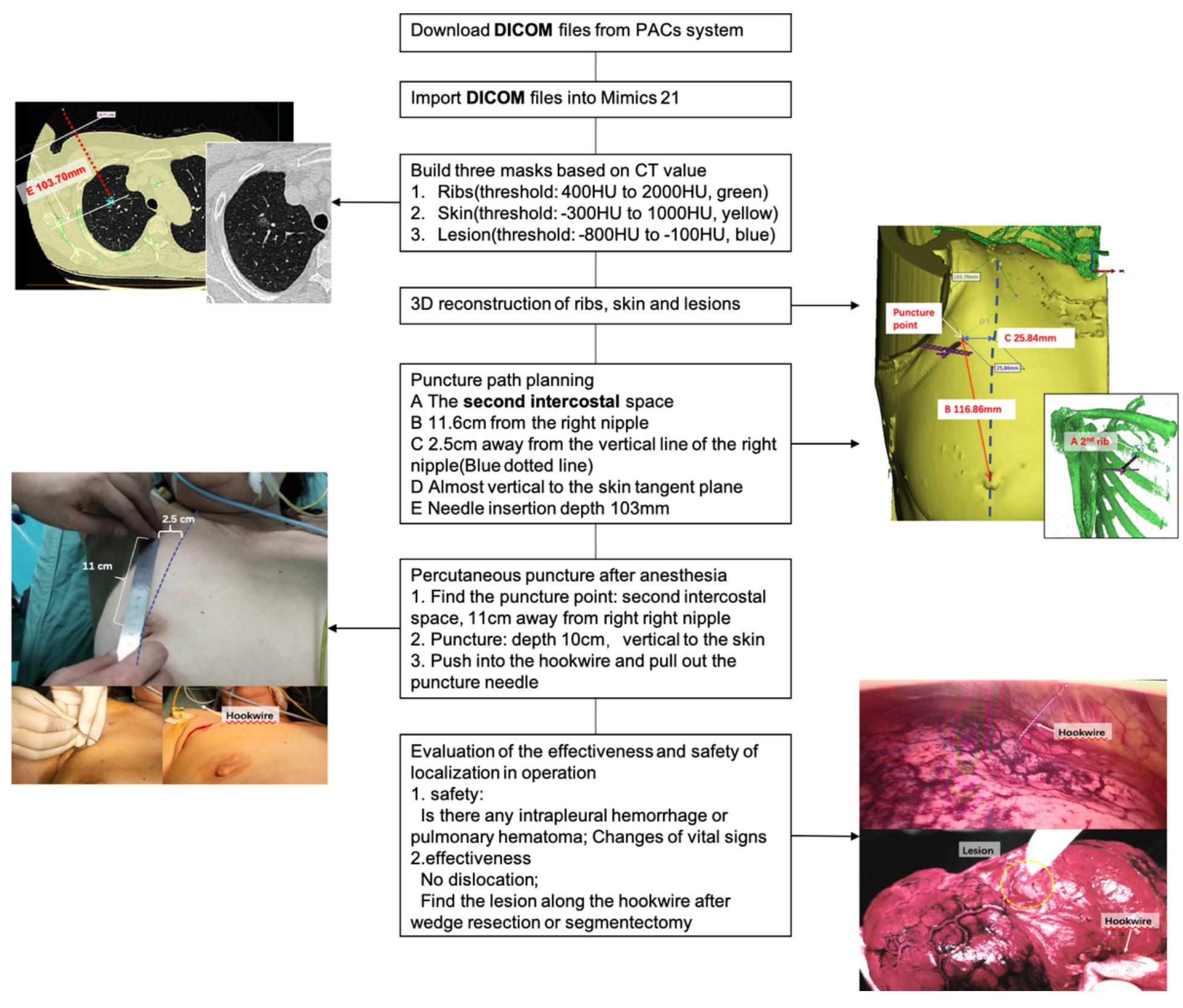

\section{Figure 1}

Flow chart of hookwire localization guided by preoperative computed tomography 3D reconstruction 